\title{
LINEAR MONOTONE OPERATORS AND WEIGHTED BMO
}

\author{
LAI QINSHENG
}

(Communicated by J. Marshall Ash)

\begin{abstract}
In this paper, the linear monotone operators, which include the well-known Hardy operator and Riemann-Liouville fractional integrals, are introduced. A necessary and sufficient condition for them to be bounded from a Banach function space into a weighted BMO is given, and their compactness in some particular cases is studied. Meanwhile, the embedding properties concerning the weighted $\mathrm{BMO}$ are investigated.
\end{abstract}

\section{INTRODUCTION}

In order to generalize the Hardy operator defined by

$$
T f(x)=\int_{0}^{x} f(t) d t, \quad x>0,
$$

many authors introduce some classes of Volterra operators and study their weighted norm inequalities. For example, in [2] Bloom and Kerman consider the operators of Hardy type defined by

$$
T f(x)=\int_{0}^{x} \phi(x, y) f(y) d y,
$$

where kernels $\phi(x, y)$ on $R^{+} \times R^{+}$with the following properties:

(i) $\phi(x, y)>0$ if $x>y$,

(ii) $\phi(x, y)$ is nondecreasing in $x$ and nonincreasing in $y$,

(iii) $\phi(x, y) \approx \phi(x, z)+\phi(z, y)$ if $y<z<x$.

The symbol " $\approx$ " means the ratio of the two sides is bounded between absolute positive constants. Simultaneously, in [10] Stepanov investigate the Volterra convolution operator $K$ given by

$$
K f(x)=\int_{0}^{x} k(x-y) f(y) d y,
$$

where the kernels $k$ satisfy the conditions:

(i) $k(x) \geq 0$ is nondecreasing on $(0, \infty)$,

(ii) $k(x+y) \leq D(k(x)+k(y))$ for all $x, y \in(0, \infty)$.

Received by the editors January 15, 1992 and, in revised form, June 30, 1992.

1991 Mathematics Subject Classification. Primary 42B25. 
The weighted norm inequalities for (2), (3) have been characterized on weighted Lebesgue spaces. In this note, we introduce a more general operator including the $T$ and $K$ above, give a necessary and sufficient condition for its boundedness from general Banach function spaces into weighted BMO, and study its compactness in some particular cases. Meanwhile, we discuss the embedding properties concerning the weighted BMO which are intrinsically interesting.

\section{BOUNDEDNESS AND COMPACTNESS}

We start with some necessary notation. Given an interval $(A, B)$ in real line $R^{1}$ (finite or infinite), let $(X, v)$ be a Banach function space on the measure space $((A, B), v d x)$, where $v(x)$ is a weight function on $(A, B)$, i.e., nonnegative locally integrable with $0<v(x)<\infty$ a.e. We omit mention of the interval $(A, B)$ concerned, since it does not cause ambiguity. The definition of Banach function spaces and their general properties can be found in $[1$, pp. 1-30], however, we would like to point out that it includes common Lebesgue spaces $L^{p} 1 \leq p \leq \infty$, Lorentz spaces, and Orlicz spaces with or without weights.

For given $(X, v)$, its associate space $\left(X^{\prime}, v\right)$ is given by

$$
\begin{aligned}
\left(X^{\prime}, v\right)= & \{g: g \text { is measurable, } \\
& \|g\|_{X^{\prime}, v}=\sup \left[\int_{A}^{B}|f g| v d x: f \in X,\|f\|_{X, v} \leq 1\right] \\
= & \left.\sup \left[\left|\int_{A}^{B} f g v d x\right|: f \in X,\|f\|_{X, v} \leq 1\right]<\infty\right\} ;
\end{aligned}
$$

$\left(X^{\prime}, v\right)$ is also a Banach function space. The following properties of Banach function spaces $(X, v)$ are needed for our argument:

$$
\begin{gathered}
0 \leq f \leq g \text { implies }\|f\|_{X, v} \leq\|g\|_{X, v}, \\
\||f|\|_{X, v}=\|f\|_{X, v} \\
\int_{A}^{B}|f g| v d x \leq\|f\|_{X, v}\|g\|_{X^{\prime}, v}
\end{gathered}
$$

for every $f \in(X, v)$ and $g \in\left(X^{\prime}, v\right)$. by

Suppose $w(x)$ is a weight function. The weighted BMO (see [6]) is defined

$$
\begin{aligned}
\mathrm{BMO}_{w}= & \{f: f \text { is locally integrable, } \\
& \left.\|f\|_{*, w}=\sup _{[a, b] \subset(A, B)} \frac{1}{b-a} \int_{a}^{b}\left|f(x)-(f)_{w,(a, b)}\right| w(x) d x<\infty\right\},
\end{aligned}
$$

where $(f)_{w,(a, b)}=(1 / w(a, b)) \int_{a}^{b} f w d x$ with $w(a, b)=\int_{a}^{b} w d x$. For a measurable set $E,|E|$ is its Lebesgue measure, $w(E)=\int_{E} w d x$, and $\chi_{E}$ denotes its characteristic function. When $w(x) \equiv 1$, we shall omit the index $w$ in the notation $\mathrm{BMO}_{w}$ and its norm. 
Let $T$ be a operator which maps the appropriate functions on $(A, B)$ into the functions on $(A, B)$. We say $T$ is monotone if $T f(x)$ is monotone whenever $f \geq 0$. For a given operator $T$, its adjoint operator $T^{*}$ is defined by

$$
\int_{A}^{B} T f(x) g(x) d x=\int_{A}^{B} f(x) T^{*} g(x) d x
$$

for all integrable $g(x)$ with compact support in $(A, B)$.

Theorem 1. Let $w, v$ be a pair of weight functions and $(X, v)$ be a Banach function space. Suppose $T$ is a linear monotone operator, and for $[a, b] \subset$ $(A, B)$ set

$$
G_{w,(a, b)}(x)=\frac{\int_{a}^{x} w-\int_{x}^{b} w}{(b-a) w(a, b)} w(x) \chi_{[a, b]}(x)
$$

Then

$$
\|T f\|_{*, w} \leq C\|f\|_{X, v}
$$

holds for all $f$ in $(X, v)$ if and only if

$$
\sup _{[a, b] \subset(A, B)}\left\|\frac{T^{*} G_{w,(a, b)}}{v}\right\|_{X^{\prime}, v}=J<\infty .
$$

Furthermore, for the best constant $C$ in (8), we have

$$
J \leq C \leq 4 J \text {. }
$$

Proof. Sufficiency. For any function $g$ and $[a, b] \subset(A, B)$, it is obvious that

$$
\begin{aligned}
& \frac{1}{b-a} \int_{a}^{b}\left|g(x)-(g)_{w,(a, b)}\right| w(x) d x \\
& \quad \leq \frac{1}{(b-a) w(a, b)} \int_{a}^{b} \int_{a}^{b}|g(x)-g(y)| w(y) w(x) d y d x \\
& \quad \leq \frac{2}{b-a} \int_{a}^{b}\left|g(x)-(g)_{w,(a, b)}\right| w(x) d x .
\end{aligned}
$$

If $g(x)$ is monotone, say increasing, then we have

$$
\begin{aligned}
\int_{a}^{b} \int_{a}^{b}|g(x)-g(y)| w(y) w(x) d y d x \\
=\int_{a}^{b} \int_{a}^{x}[g(x)-g(y)] w(y) w(x) d y d x \\
\quad+\int_{a}^{b} \int_{x}^{b}[g(y)-g(x)] w(y) w(x) d y d x \\
=\int_{a}^{b} g(x) w(x)\left[\int_{a}^{x} w-\int_{x}^{b} w\right] d x \\
\quad+\int_{a}^{b}\left[\int_{x}^{b} g w-\int_{a}^{w} g w\right] w(x) d x \\
=2 \int_{a}^{b} g(x) w(x)\left[\int_{a}^{x} w-\int_{x}^{b} w\right] d x .
\end{aligned}
$$


Similarly, we get

$$
\begin{aligned}
\int_{a}^{b} & \int_{a}^{b}|g(x)-g(y)| w(y) w(x) d y d x \\
& =-2 \int_{a}^{b} g(x) w(x)\left[\int_{a}^{x} w-\int_{x}^{b} w\right] d x
\end{aligned}
$$

when $g$ is decreasing.

Combining (14)-(16) and (10), we have, for $f \geq 0$,

$$
\begin{aligned}
\frac{1}{b-a} & \int_{a}^{b}\left|T f(x)-(T f)_{w,(a, b)}\right| w(x) d x \\
& \leq 2\left|\int_{A}^{B} T f(x) G_{w,(a, b)}(x) d x\right|=2\left|\int_{A}^{B} f(x) T^{*} G_{w,(a, b)}(x) d x\right| \\
& \leq \frac{2}{b-a} \int_{a}^{b}\left|T f(x)-(T f)_{w,(a, b)}\right| w(x) .
\end{aligned}
$$

It follows from the Hölder inequality (7) that

$$
\left|\int_{A}^{B} f(x) T^{*} G_{w,(a, b)}(x) d x\right| \leq\|f\|_{X, v}\left\|\frac{T^{*} G_{w,(a, b)}}{v}\right\|_{X^{\prime}, v} \leq J\|f\|_{X, v} .
$$

Therefore, for $f \geq 0, f \in(X, v)$, we obtain

$$
\|T f\|_{*, w} \leq 2 J\|f\|_{X^{\prime}, v} .
$$

For general $f \in(X, v)$, write $f=f^{+}-f^{-}$, where $f^{+}(x)=\max \{0, f(x)\}$. It follows that

$$
\begin{aligned}
\|T f\|_{*, w} & \leq\left\|T\left(f^{+}\right)\right\|_{*, w}+\left\|T\left(f^{-}\right)\right\|_{*, w} \\
& \leq 2 J\left(\left\|f^{+}\right\|_{X, v}+\left\|f^{-}\right\| X, v\right) \leq 4 J\|f\|_{X, v} .
\end{aligned}
$$

Conversely, assume $\|T f\|_{*, w} \leq C\|f\|_{X, v}$. For any $[a, b] \subset(A, B)$, it follows from (17) that

$$
\left|\int_{A}^{B} f(x) T^{*} G_{w,(a, b)}(x) d x\right| \leq C\|f\|_{X, v}
$$

for all $f \geq 0, f \in(X, v)$. Taking supremum over all $f \geq 0,\|f\|_{X, v} \leq 1$, one obtains

$$
\left\|\frac{T^{*} G_{w,(a, b)}}{v}\right\|_{X^{\prime}, v} \leq C ;
$$

hence $J \leq C$. The proof of Theorem 1 is complete.

It is obvious that the operators given by (2) and (3) both are linear monotone operators, and the special case of Theorem 1 , when $T$ is the Hardy operator defined by (1), has been obtained in [4].

Theorem 2. Suppose $T$ is a linear monotone operator satisfying that

$$
T f(x) \equiv 0 \text { on }(A, R] \text { whenever supp } f \subset[R, B) \quad(R \in(A, B)) \text {. }
$$


If the operator $P_{R} f=T\left(f \chi_{(A, R]}\right)$ is compact from $(X, v)$ into $\mathrm{BMO}_{w}$ for every $R \in(A, B)$, then $T$ is compact from $(X, v)$ into $\mathrm{BMO}_{w}$ if and only if both (12) and

$$
J_{R}=\sup _{[a, b] \subset(A, B)}\left\|\frac{T^{*} G_{w,(a, b)}}{v} \chi_{(R, B)}\right\|_{X^{\prime}, v} \rightarrow 0 \text { as } R \rightarrow B .
$$

hold.

Proof. Sufficiency. Let $T_{R} f=T\left(f \chi_{(R, B)}\right)$. Then $T=P_{R}+T_{R}$. Since also $T_{R}$ is a linear monotone operator from $(X, v)$ into $\mathrm{BMO}_{w}$ and $T_{R}^{*} g=\chi_{(R, B)} T^{*} g$, it follows from Theorem 1 that

$$
\left\|T_{R}\right\| \leq 4 J_{R} \rightarrow 0 \text { as } R \rightarrow B .
$$

Therefore, $T$ is compact from $(X, v)$ into $\mathrm{BMO}_{w}$.

Necessity. We only need to verify (19), if (19) is not true, then there exist an $\varepsilon>0, R_{n} \rightarrow B$, and $\left[a_{n}, b_{n}\right]$, such that

$$
\left\|\frac{T^{*} G_{w,\left(a_{n}, b_{n}\right)}}{v} \chi_{\left(R_{n}, B\right)}\right\|_{X^{\prime}, v} \geq \varepsilon ;
$$

therefore, for every $n$, there is a $g_{n} \geq 0$ with $\left\|g_{n}\right\|_{X, v} \leq 1$ such that

$$
\left|\int_{a_{n}}^{b_{n}} T^{*} G_{w,\left(a_{n}, b_{n}\right)}(x) \chi_{\left(R_{n}, B\right)}(x) g_{n}(x) d x\right|>\frac{\varepsilon}{2} .
$$

Let $f_{n}(x)=g_{n}(x) \chi_{\left[a_{n}, b_{n}\right]}(x) \chi_{\left(R_{n}, B\right)}(x)$. Then $\left\|f_{n}\right\|_{X, v} \leq 1$. Select a subsequence from $\left\{f_{n}\right\}$ by induction: $n_{1}=1$, and $n_{k+1}$ is chosen such that $R_{n_{k+1}}>\max \left\{R_{n_{k}}, b_{n_{k}}\right\}(k=1,2, \ldots)$.

For every pair $k<s$, observing that $R_{n_{s}}>b_{n_{k}}$ and $f_{n_{s}} \equiv 0$ on $\left(A, R_{n_{s}}\right)$, it follows from (18), (17), and (20) that

$$
\begin{aligned}
& \left.\left\|T f_{n_{k}}-T f_{n_{s}}\right\|_{*, w} \geq \frac{1}{b_{n_{k}}-a_{n_{k}}} \int_{a_{n_{k}}}^{b_{n_{k}}} \mid T f_{n_{k}}(x)-\left(T f_{n_{k}}\right)_{w,\left(a_{n_{k}}, b_{n_{k}}\right)}\right) w(x) d x \\
& \geq\left|\int_{a_{n_{k}}}^{b_{n_{k}}} T^{*} G_{w,\left(a_{n_{k}}, b_{n_{k}}\right)}(x) f_{n_{k}}(x) d x\right|>\frac{\varepsilon}{2},
\end{aligned}
$$

which implies that $T$ is not compact from $(X, v)$ into $\mathrm{BMO}_{w}$. This completes Theorem 2.

The first important example of linear monotone operators is the RiemannLiouville fractional integrals (see $[5,9])$ defined by

$$
T_{\alpha} f(x)=\frac{1}{\Gamma(\alpha)} \int_{0}^{x}(x-t)^{\alpha-1} f(t) d t, \quad x>0, \alpha \geq 1,
$$

and then

$$
T_{\alpha}^{*} g(x)=\frac{1}{\Gamma(\alpha)} \int_{x}^{\infty}(t-x)^{\alpha-1} g(t) d t .
$$

When $\alpha=n$ is a positive integer, we have

$$
T_{1}^{*} G_{w,(a, b)}(x)=G_{w,(a, b)}^{1}(x)=\frac{w(a, x) w(x, b)}{(b-a) w(a, b)} \chi_{[a, b]}(x)
$$


and

$$
T_{n}^{*} G_{w,(a, b)}(x)=G_{w,(a, b)}^{n}(x)=\int_{x}^{b} G_{w,(a, b)}^{n-1}(t) d t \quad(n \geq 2) .
$$

Furthermore, if $w(x) \equiv 1$, a simple calculation shows that (24)

$$
G_{1,(a, b)}^{n}(x)=\left\{\begin{array}{cc}
0, & x \in(b, \infty), \\
\frac{(b-x)^{n}[2 x-(n+1) a+(n-1) b]}{(n+1) !(b-a)^{2}}, & x \in[a, b], \\
\frac{1}{(n+1) !(b-a)^{2}}\left\{(b-x)^{n}[2 x-(n+1) a+(n-1) b]\right. \\
\left.-(a-x)^{n}[2 x+(n-1) a-(n+1) b]\right\}, \\
x \in(0, a) .
\end{array}\right.
$$

In this case, Theorem 1 can be rewritten as follows (cf. [7]).

Theorem $\mathbf{1}^{\prime}$. Let $A C^{(n-1)}(A, B)=\left\{f: f^{(n-1)}\right.$ is absolutely continuous in $(A, B)$, and $\left.f(A)=f^{\prime}(A)=\cdots=f^{(n-1)}(A)=0\right\}$. Suppose the set $\left\{f^{(n)}: f \in\right.$ $\left.A C^{(n-1)}\right\}$ is dense in $(X, v)$. Then

$$
\|f\|_{*, w} \leq C\left\|f^{(n)}\right\|_{X, v}
$$

holds for all $f \in A C^{(n-1)}$ if and only if

$$
\sup _{[a, b] \subset(A, B)}\left\|\frac{G_{w,(a, b)}^{n}}{v}\right\|_{X^{\prime}, v}=J<\infty .
$$

Moreover, for the best constant $C$, there is the estimate $J \leq C \leq 4 J$.

It is obvious that Theorem $1^{\prime}$ gives some sufficient conditions such that the weighted Sobolev space concerning $(X, v)$ is continuously embedded into $\mathrm{BMO}_{w}$. We shall omit it.

The other example is a Volterra operator given by

$$
T f(x)=\delta(x) \int_{0}^{x} f(t) \sigma(t) d t, \quad x>0,
$$

where $\delta(x)$ and $\sigma(x)$ are prescribed functions satisfying local integrability and $\frac{\sigma}{v} \in\left(X^{\prime}, v\right)$ respectively (see [3]). It is obvious that

$$
T^{*} g(x)=\sigma(x) \int_{x}^{\infty} g(t) \delta(t) d t
$$

In addition, we assume $\delta$ and $\sigma$ are weight functions and $\delta$ is increasing. Then $T$ is a linear monotone operator. Applying Theorem 2 to this operator, we obtain a criterion for its compactness.

A Banach function space (or a normed function space) $(X, v)$ is said to have absolutely continuous norm if, for every $f \in(X, v)$ and any sequence of measurable sets $\left\{E_{n}\right\}$ with $E_{n} \rightarrow \varnothing$ a.e., the equation

$$
\lim _{n \rightarrow \infty}\left\|f \chi_{E_{n}}\right\|_{X, v}=0
$$

holds. 
Theorem 3. Suppose $T$ is defined as above and $\delta$ is bounded. Assume $\left(X^{\prime}, v\right)$ has absolutely continuous norm and $\|w\|_{\infty}<\infty$. Then $T$ is compact from $(X, v)$ into $\mathrm{BMO}_{w}$ if and only if

(i)

and

$$
J=\sup _{[a, b] \subset(0, \infty)}\left\|\frac{T^{*} G_{w,(a, b)}}{v}\right\|_{X^{\prime}, v}<\infty
$$

(ii)

$$
J_{R}=\sup _{[a, b] \subset(0, \infty)}\left\|\frac{T^{*} G_{w,(a, b)}}{v} \chi_{(R, \infty)}\right\|_{X^{\prime}, v} \rightarrow 0, \quad \text { as } R \rightarrow \infty \text {. }
$$

Proof. According to Theorem 2, it is sufficient to prove that $T_{R} f=T\left(f \chi_{(0, R]}\right)$ is compact from $(X, v)$ into $\mathrm{BMO}_{w}$ for every given $R>0$. Indeed, suppose $\left\{f_{n}\right\}$ is a sequence in the closed unit ball of $(X, v)$, we have

$$
\left|T_{R} f_{n}(x)\right| \leq \delta(R)\left\|\frac{\sigma}{v}\right\|_{X^{\prime}, v} \text { for any } n \text { and } x \in[0, R]
$$

and

$$
\left|T_{R} f_{n}(x)-T_{R} f_{n}(y)\right| \leq \delta(R)\left\|\frac{\sigma}{v} \chi_{[x, y]}\right\|_{X^{\prime}, v}
$$

for any $n$ and $0 \leq x<y \leq R$. Since $\frac{\sigma}{v} \in\left(X^{\prime}, v\right)$ and $\left(X^{\prime}, v\right)$ has absolutely continuous norm, $\left\{T_{R} f_{n}\right\}$ is uniformly bounded and equicontinuous. By use of the Arzela-Ascoli theorem it contains a subsequence which is uniformly convergent on $[0, R]$ and, furthermore, on $[0, \infty)$, because $T_{R} f_{n}(x)=$ $\delta(x) \int_{0}^{R} f_{n}(t) \sigma(t) d t$ on $(R, \infty)$. Note that

$$
\|f\|_{*, w} \leq 2\|w\|_{\infty}\|f\|_{\infty},
$$

if $\|w\|_{\infty}<\infty$; the corresponding subsequence is convergent in $\mathrm{BMO}_{w}$. The proof of Theorem 3 is complete.

When $\delta(x)=\sigma(x)=1$, the previous $T$ is the Hardy operator defined in (1). For the Hardy operator, we can remove the prescribed condition $\sigma / v=$ $1 / v \in\left(X^{\prime}, v\right)$ in Theorem 3. Indeed, we have

Theorem 4. Suppose $T$ is the Hardy operator defined in (1). Assume $\left(X^{\prime}, v\right)$ has absolutely continuous norm and $\|w\|_{\infty}<\infty$. Then, $T$ is compact from $(X, v)$ into $\mathrm{BMO}_{w}$ if and only if

(i)

(ii)

$$
J=\sup _{[a, b] \subset(0, \infty)}\left\|\frac{G_{w,(a, b)}^{1}}{v}\right\|_{X^{\prime}, v}<\infty,
$$

$$
J_{R}=\sup _{[a, b] \subset(0, \infty)}\left\|\frac{G_{w,(a, b)}^{1}}{v} \chi_{(R, \infty)}\right\|_{X^{\prime}, v} \rightarrow 0, \quad \text { as } R \rightarrow \infty \text {, }
$$

and

(iii)

$$
J_{r}=\sup _{[a, b] \subset(0, \infty)}\left\|\frac{G_{w,(a, b)}^{1}}{v} \chi_{(0, r)}\right\|_{X^{\prime}, v} \rightarrow 0, \quad \text { as } r \rightarrow 0,
$$

where $G_{w,(a, b)}^{1}(x)$ is defined in (22). 
Proof. Sufficiency. It is enough to prove that, for given $R>0, T_{R}$ is compact. For any $0<r<R$, write $T_{R}=P_{R, r}+P_{r}$ with $P_{r} f=T\left(f \chi_{(0, r)}\right)$ and $P_{R, r} f=$ $T\left(f \chi_{[r, R]}\right)$. Since $J<\infty$ implies $\chi_{[r, R]} / v \in\left(X^{\prime}, v\right)$ for any $0<r<R<\infty$, the argument similar to that used in Theorem 3 shows $P_{R, r}$ is compact. On the other hand we have $\left\|P_{r}\right\| \leq 4 J_{r}$; therefore, $T_{R}$ is compact.

Necessity. We only need to verify (iii). The procedure is nearly the same as that in Theorem 2 for proving that the compactness of $T$ implies (19). We shall only mention the difference. In this case, we select subsequence by $n_{1}=1$, and $n_{k+1}$ is chosen such that $r_{n_{k+1}}<\min \left\{r_{n_{k}}, a_{n_{k}}\right\}(k=1,2, \ldots)$. Then, for every $k<s, T f_{n_{s}}(x) \equiv C_{n_{s}}=\int_{0}^{b_{n_{s}}} f_{n_{s}}(t) d t$ on $\left[a_{n_{k}}, b_{n_{k}}\right]$. Hence, it follows that

$$
\begin{aligned}
& \left\|T f_{n_{k}}-T f_{n_{s}}\right\|_{*, w} \\
& \quad \geq \frac{1}{b_{n_{k}}-a_{n_{k}}} \int_{a_{n_{k}}}^{b_{n_{k}}}\left|T\left(f_{n_{k}}-f_{n_{s}}\right)(x)-\left(T\left(f_{n_{k}}-f_{n_{s}}\right)\right)_{w,\left(a_{n_{k}}, b_{n_{k}}\right)}\right| w(x) d x \\
& \quad=\frac{1}{b_{n_{k}}-a_{n_{k}}} \int_{a_{n_{k}}}^{b_{n_{k}}}\left|T f_{n_{k}}(x)-\left(T f_{n_{k}}\right)_{w,\left(a_{n_{k}}, b_{n_{k}}\right)}\right| w(x) d x \\
& \quad \geq\left|\int_{a_{n_{k}}}^{b_{n_{k}}} T^{*} G_{w,\left(a_{n_{k}}, b_{n_{k}}\right)}(x) f_{n_{k}}(x) d x\right|>\frac{\varepsilon}{2} .
\end{aligned}
$$

The proof of Theorem 4 is complete.

A function $f$ in a normed function space $X$ is said to have the continuous norm if for every $x \in(A, B)$ and $\varepsilon>0$ given there exists a $\delta>0$ such that $\left\|f \chi_{[x-r, x+r]}\right\|_{X}<\varepsilon$, whenever $0<r<\delta$.

In [4] we proved that, if Hardy operator is compact from a Banach function space $(X, v)$ into BMO, then for every given $0<r<R<\infty$ the function $\chi_{[r, R]} / v$ has continuous norm in $\left(X^{\prime}, v\right)$. Thus, the argument in Theorem 4 shows:

Theorem $4^{\prime}$. Suppose $(X, v)$ is a Banach function space. Then the Hardy operator is compact from $(X, v)$ into $B M O$ if and only if:

(i)

$$
J=\sup _{[a, b] \subset(0, \infty)}\left\|\frac{G_{1,(a, b)}^{1}}{v}\right\|_{X^{\prime}, v}<\infty ;
$$

(ii) for every given $0<r<R<\infty$, the function $\chi_{[r, R]} / v$ has continuous norm in $\left(X^{\prime}, v\right)$;

(iii)

$$
J_{r}=\sup _{[a, b] \subset(0, \infty)}\left\|\frac{G_{1,(a, b)}^{1}}{v} \chi_{(0, r)}\right\|_{X^{\prime}, v} \rightarrow 0, \quad \text { as } r \rightarrow 0,
$$

and

$$
J_{R}=\sup _{[a, b] \subset(0, \infty)}\left\|\frac{G_{1,(a, b)}^{1}}{v} \chi_{(R, \infty)}\right\|_{X^{\prime}, v} \rightarrow 0, \quad \text { as } R \rightarrow \infty,
$$

where $G_{1,(a, b)}^{1}(x)=(b-x)(x-a) \chi_{[a, b]}(x) /(b-a)^{2}$ is defined in (22) with $w(x) \equiv 1$. 
Theorems 4 and $4^{\prime}$ are the improved versions of our previous compactness criteria in [4].

\section{EMBEDDING PROPERTIES CONCERNING WEIGHTED BMO}

In Theorems 3 and 4 , the condition $\|w\|_{\infty}<\infty$ just guarantee the inequality (25). Hence, when we try to generalize this condition, a natural problem arises, that is, whether $\|w\|_{\infty}<\infty$ is necessary for $\|f\|_{*}, w \leq C\|f\|_{\infty}$. More generally, given a function space $X$, under which condition is $X$ is continuously embedded into $\mathrm{BMO}_{w}$ ? And how about the inverse embedding? The following theorems treat these questions.

Theorem 5. The inequality $\|f\|_{*}, w \leq C\|f\|_{\infty}$ holds for all $f \in L_{\infty}$, if and only if $\|w\|_{\infty}<\infty$. Furthermore, for the best constant $C,\|w\|_{\infty}=C$.

Proof. Sufficiency. Suppose $\|w\|_{\infty}<\infty$. We shall prove $\|f\|_{*, w} \leq\|w\|_{\infty}$ for all $f \in L_{\infty}$ with $\|f\|_{\infty} \leq 1$.

For every interval $[a, b] \subset(A, B)$ given, without loss of generality, we may assume $(f)_{w,(a, b)} \geq 0$. Otherwise, we consider $-f$. Then $(-f)_{w,(a, b)} \geq 0$ and

$$
\int_{a}^{b}\left|f(x)-(f)_{w,(a, b)}\right| w(x) d x=\int_{a}^{b}\left|-f(x)-(-f)_{w,(a, b)}\right| w(x) d x
$$

Write $f=f^{+}-f^{-}$, where $f^{+}=\max \{f(x), 0\}$. Let $E_{1}=\{x \in[a, b]$ : $\left.f(x) \geq(f)_{w,(a, b)}\right\}, E_{2}=\left\{x \in[a, b]: 0 \leq f(x) \leq(f)_{w,(a, b)}\right\}$, and $E_{3}=\{x \in$ $[a, b]: f(x)<0\}$. Then

$$
\begin{aligned}
& \int_{a}^{b}\left|f(x)-(f)_{w,(a, b)}\right| w(x) d x \\
& =\int_{E_{1}}\left(f^{+}-(f)_{w,(a, b)}\right) w d x \\
& \quad+\int_{E_{2}}\left((f)_{w,(a, b)}-f^{+}\right) w d x+\int_{E_{3}}\left(f^{-}+(f)_{w,(a, b)}\right) w d x
\end{aligned}
$$

If $w\left(E_{2}\right)+w\left(E_{3}\right) \leq w\left(E_{1}\right)$, it follows from (26) that

$$
\begin{aligned}
\int_{a}^{b}\left|f(x)-(f)_{w,(a, b)}\right| w(x) d x \\
=\int_{E_{1}} f^{+} w d x+\int_{E_{2}} f^{+} w d x+\int_{E_{3}} f^{-} w d x \\
\quad+\left(w\left(E_{2}\right)+w\left(E_{3}\right)-w\left(E_{1}\right)\right)(f)_{w,(a, b)}-2 \int_{E_{2}} f^{+} w d x \\
\leq \int_{E_{1}} f^{+} w d x+\int_{E_{2}} f^{+} w d x+\int_{E_{3}} f^{-} w d x \\
=\int_{a}^{b}|f(x)| w(x) d x \leq\|w\|_{\infty}(b-a)
\end{aligned}
$$


When $w\left(E_{2}\right)+w\left(E_{3}\right)>w\left(E_{1}\right)$, the right side of $(26)$ is

$$
\begin{aligned}
\int_{E_{1}} f^{+} & w d x-\int_{E_{2}} f^{+} w d x \\
& +\int_{E_{3}} f^{-} w d x+w(a, b)(f)_{w,(a, b)}-2 w\left(E_{1}\right)(f)_{w,(a, b)} \\
\leq & \int_{E_{1}} f^{+} w d x-\int_{E_{2}} f^{+} w d x+\int_{E_{3}} f^{-} w d x+\int_{a}^{b} f w d x \\
= & 2 \int_{E_{1}} f^{+} w d x \leq 2 w\left(E_{1}\right) \leq w(a, b) \leq\|w\|_{\infty}(b-a) .
\end{aligned}
$$

Combining (27) and (28), we have $\|f\|_{*, w} \leq\|w\|_{\infty}$, whenever $\|f\|_{\infty} \leq 1$.

Necessity. We need to prove that $\|f\|_{*, w} \leq C\|f\|_{\infty}$ implies $\|w\|_{\infty}$ $\leq C$. Assume $\|w\|_{\infty}>C$. Then there exists $\varepsilon>0$ such that

$$
|E|=|\{x \in(A, B): w(x)>(1+2 \varepsilon) C\}|>0 .
$$

On the other hand, there is a $[a, b] \subset(A, B)$ satisfying

$$
|[a, b] \cap E|>\frac{b-a}{1+\varepsilon} \text {. }
$$

We can divide $[a, b] \cap E$ into two disjoint subsets $E_{i}(i=1,2)$ with $w\left(E_{1}\right)=$ $w\left(E_{2}\right)$. Let

$$
f(x)=\chi_{E_{1}}(x)-\chi_{E_{2}}(x) .
$$

Then $f_{w,(a, b)}=0$, and

$$
\begin{aligned}
\|f\|_{*, w} & \geq \frac{1}{b-a} \int_{a}^{b}\left|f(x)-(f)_{w,(a, b)}\right| w(x) d x \\
& =\frac{w\left(E_{1} \cup E_{2}\right)}{b-a} \geq \frac{1+2 \varepsilon}{1+\varepsilon} C>C\|f\|_{\infty} .
\end{aligned}
$$

This is a contradiction. The proof of Theorem 5 is complete.

Theorem 5 gives not only a necessary and sufficient condition for $L_{\infty}$ to be continuously embedded into $\mathrm{BMO}_{w}$ but also the exact estimate for the best constant $C$, which improves the well-known estimate (25). Replacing $L_{\infty}$ by BMO, we have

Theorem 6. The inequality $\|f\|_{*, w} \leq C\|f\|_{*}$ holds for all $f \in \mathrm{BMO}$, if and only if $\|w\|_{\infty}<\infty$. Moreover, for the best constant $C,\|w\|_{\infty} \leq C \leq 2\|w\|_{\infty}$. Proof. It is sufficient to prove that $\|f\|_{*, w} \leq C\|f\|_{*}$ implies $\|w\|_{\infty} \leq C$. Assume $\|w\|_{\infty}>C$. Then there exists $0<\varepsilon<1$ such that

$$
|E|=|\{x \in(A, B): w(x)>C /(1-\varepsilon)\}|>0 .
$$

Meanwhile, there is a $[a, b] \subset(A, B)$ satisfying

$$
|[a, b] \cap E|>(1-\varepsilon)(b-a) .
$$

We can divide $[a, b] \cap E$ into two disjoint subsets $E_{i}(i=1,2)$ with equal measure. If $w\left(E_{1}\right) \leq w\left(E_{2}\right)$, let $f(x)=\chi_{E_{1}}(x)$. Then, noting that $\left\|\chi_{E}\right\|_{*}=\frac{1}{2}$ 
for any measurable set $E \subsetneq(A, B)$ with positive measure (see [8]), it follows that

$$
\begin{aligned}
\|f\|_{*, w} & \geq \frac{1}{b-a} \int_{E_{1} \cup E_{2}}\left|f(x)-(f)_{w,(a, b)}\right| w(x) d x \\
& =\frac{w\left(E_{1}\right)}{b-a}+\frac{w\left(E_{1}\right)}{(b-a) w(a, b)}\left(w\left(E_{2}\right)-w\left(E_{1}\right)\right) \\
& \geq \frac{w\left(E_{1}\right)}{b-a}>C\|f\|_{*} .
\end{aligned}
$$

If $w\left(E_{1}\right)>w\left(E_{2}\right)$, let $f(x)=\chi_{E_{2}}(x)$. A similar argument shows that

$$
\|f\|_{*, w} \geq \frac{w\left(E_{2}\right)}{b-a}+\frac{w\left(E_{2}\right)}{(b-a) w(a, b)}\left(w\left(E_{1}\right)-w\left(E_{2}\right)\right)>C\|f\|_{*} .
$$

This is a contradiction. The proof of Theorem 6 is complete.

Conversely, we have

Theorem 7. The inequality $\|f\|_{*} \leq C\|f\|_{*, w}$ holds for all $f \in \mathrm{BMO}_{w}$, if and only if $\left\|\frac{1}{w}\right\|_{\infty}<\infty$. And, for the best constant $C$,

$$
\frac{1}{4}\left\|\frac{1}{w}\right\|_{\infty} \leq C \leq 2\left\|\frac{1}{w}\right\|_{\infty} .
$$

Proof. It is sufficient to prove that $\|f\|_{*} \leq C\|f\|_{*, w}$ implies $\frac{1}{4}\left\|\frac{1}{w}\right\|_{\infty} \leq C$. Assume it is not true. Then there exists an $\varepsilon>0$ such that

$$
|E|=|\{x \in(A, B): 1 / w(x)>4 C+\varepsilon\}|>0 .
$$

Let $f(x)=\chi_{E}(x)$. Then

$$
\begin{aligned}
& \frac{1}{b-a} \int_{a}^{b}\left|f(x)-(f)_{w,(a, b)}\right| w(x) d x \\
& \quad=\frac{2 w([a, b] \cap E) w\left([a, b] \cap E^{c}\right)}{(b-a) w(a, b)} \leq \frac{2 w([a, b] \cap E)}{(b-a)} \leq \frac{2}{4 C+\varepsilon} .
\end{aligned}
$$

Therefore,

$$
\frac{1}{2}=\|f\|_{*} \leq C\|f\|_{*, w} \leq \frac{2 C}{4 C+\varepsilon} .
$$

This contradiction completes the proof of Theorem 7 .

Combining Theorems 6 and 7 shows that $\mathrm{BMO}$ is equivalent to $\mathrm{BMO}_{w}$ if and only if the weight function is bounded above and bounded away from zero. However, usually, $\mathrm{BMO}_{w}$ cannot be continuously embedded into another function space, if $\|1\|_{X}>0$. Conversely, we have

Theorem 8. Suppose $X$ is a linear normed space which consists of functions on $(A, B)$ and contains all simple functions. If $X$ has absolutely continuous norm (see the definition before Theorem 3), then $X$ is not continuously embedded into $\mathrm{BMO}_{w}$ except $w(x) \equiv 0$ a.e.

Proof. Suppose $w(x) \not \equiv 0$. Let $I(x, r)=[x-r, x+r]$. According to Lebesgue differential theorem, there exists $x_{0} \in(A, B)$, such that

$$
\lim _{r \rightarrow 0} \frac{1}{\left|I\left(x_{0}, r\right)\right|} \int_{I\left(x_{0}, r\right)} w(x) d x=w\left(x_{0}\right)>0
$$


For every $I\left(x_{0}, r\right)$, choose $c_{r}$ satisfying $w\left(x_{0}-r, c_{r}\right)=w\left(c_{r}, x_{0}+r\right)=$ $\frac{1}{2} w\left(I\left(x_{0}, r\right)\right)$. Let

$$
f_{r}(x)=\chi_{\left[x_{0}-r, c_{r}\right]}(x)-\chi_{\left(c_{r}, x_{0}+r\right]}(x) .
$$

Then $\left\|f_{r}\right\|_{X} \rightarrow 0$ as $r \rightarrow 0$. However, since $\left(f_{r}\right)_{w, I\left(x_{0}, r\right)}=0$, we have

$$
\begin{aligned}
\left\|f_{r}\right\|_{*, w} & \geq \frac{1}{\left|I\left(x_{0}, r\right)\right|} \int_{I\left(x_{0}, r\right)} \mid f_{r}(x)-\left(f_{r}\right)_{w, I\left(x_{0}, r\right) \mid w(x) d x} \\
& =\frac{1}{I\left(x_{0}, r\right) \mid} \int_{I\left(x_{0}, r\right)} w(x) d x \rightarrow w\left(x_{0}\right)>0 \quad(\text { as } r \rightarrow 0) .
\end{aligned}
$$

The proof of Theorem 8 is complete.

Remark. The other weighted BMO is defined by

$$
\begin{aligned}
\mathrm{BMO}^{w}= & \{f: f \text { is locally integrable, } \\
& \left.\|f\|_{*}^{w}=\sup _{[a, b] \subset(A, B)} \frac{1}{w(a, b)} \int_{a}^{b}\left|f(x)-(f)_{(a, b)}\right| d x<\infty\right\},
\end{aligned}
$$

which corresponds to the weighted $L_{\infty}$ defined by

$$
L_{\infty}^{w}=\left\{f: f \text { is measurable, }\|f\|_{\infty}^{w}=\|f / w\|_{\infty}<\infty\right\} .
$$

All arguments in this paper are still available if $\mathrm{BMO}_{w}$ is replaced by $\mathrm{BMO}^{w}$. However, we would like to point out that the estimates for the best constants in Theorems 6 and 7 will be improved. That is, we have

Theorem 6'. The inequality $\|f\|_{*}^{w} \leq C\|f\|_{*}$ holds for all $f \in \mathrm{BMO}$, if and only if $\|1 / w\|_{\infty}<\infty$. Moreover, the best constant $C$ is equal to $\|1 / w\|_{\infty}$.

Theorem 7'. The inequality $\|f\|_{*} \leq C\|f\|_{*}^{w}$ holds for all $f \in \mathrm{BMO}^{w}$, if and only if $\|w\|_{\infty}<\infty$. And, for the best constant $C, \frac{1}{4}\|w\|_{\infty} \leq C \leq\|w\|_{\infty}$.

On the other hand, all theorems in $\S 3$ hold also in more general cases, for example, in high-dimension Euclidean spaces.

\section{ACKNOWLEDGMENT}

The author wishes to express many thanks to Professor W. D. Evans and Dr. J. R. Partington for a lot of helpful conversations and suggestions.

\section{REFERENCES}

1. C. Bennet and R. Sharpley, Interpolation of operators, Academic Press, New York, 1988.

2. S. Bloom and R. Kerman, Weighted norm inequalities for operators of Hardy type, Proc. Amer. Math. Soc. 113 (1991), 135-141.

3. D. E. Edmunds, W. D. Evans, and D. J. Harris, Approximation numbers of certain Volterra integral operators, J. London Math. Soc. (2) 37 (1988), 471-489.

4. Lai Qinsheng and L. Pick, The Hardy operator, $L_{\infty}$, and BMO, J. London Math. Soc. (2) (to appear).

5. F. Martin-Reyes and E. Sawyer, Weighted norm inequalities for the Riemann-Liouville fractional integral operators of order one and greater, Proc. Amer. Math. Soc. 106 (1989), 727733. 
6. B. Muckenhoupt and R. L. Wheeden, Weighted bounded mean oscillation and the Hilber transform, Studia Math. 54 (1976), 221-237.

7. B. Opic and A. Kufner, Hardy-type inequalities, Longman, Harlow, 1990.

8. U. Neri, Some properties of functions with bounded mean oscillation, Studia Math. 61 (1977), 63-75.

9. V. D. Stepanov, Two-weighted estimates for Riemann-Liouville integrals, Ceskoslovenska Akad. Vid. 39 (1988), 1-28.

10. __, Weighted inequalities for a class of Volterra convolution operators, J. London Math. Soc. (2) (to appear).

Department of Pure Mathematics, The University of Leeds, Leeds, LS2 9JT England E-mail address: pmt5lq@uk.ac.leeds.sun 